\title{
Realistic Elbow Flesh Deformation Based on Anthropometrical Data for Ergonomics Modeling
}

\author{
Setia Hermawati and Russell Marshall \\ Dept. Design \& Technology \\ Loughborough University, Leicestershire \\ LE11 3TU, UK \\ \{S.Hermawati, R.Marshall\}@lboro.ac.uk
}

\begin{abstract}
The human model for ergonomic simulation has improved in terms of its reliability and appearance and yet there seems to be less attention paid to create a realistic and accurate flesh deformation around the joint. This study, a part of ongoing research, proposes a combination of manual and automatic (3D body scanner) measurements to create a database for flesh deformation prediction i.e. flesh deformation area and cross section changes, around the elbow joint. The database consists of two race groups i.e., Caucasian and Asian (23 subjects, 11 males and 12 females), which were carefully chosen to represent a variety of height and body type. The prediction results for both flesh deformation area and cross section changes are discussed as well as their relevance for the next stage of the study.
\end{abstract}

Keywords: Flesh deformation modeling, 3D body scanner, ergonomics.

\section{Introduction}

A common usage of human models in Ergonomics applications is to evaluate a product or workplace to accommodate people with diverse sizes and shapes. Locket et al. [1] pointed out that ergonomic human modeling tools were also used for visualization. The visualization provided information about body posture, reach ability, field of view and clearances which served as a basis for ergonomics evaluation and decision making [2]. A study by Lämkull et al. [3] showed that the visualization fidelity did affect the user effectiveness in engaging with ergonomics applications. They also argued that the request for a more human-like virtual human was present. This request was partly fulfilled by the recent development of Vis Jack which allows the incorporation of $3 \mathrm{D}$ body scanner data to generate a more accurate and realistic virtual human. Despite this latest development, there seems to be a lack of attention paid to the development of a realistic and yet accurate flesh deformation around the joint which would be useful to address motion restriction due to clothes, gloves, etc.

\subsection{Related Studies}

A review of flesh deformation methods revealed a number of different approaches. The most realistic, but complicated and computationally demanding, was anatomic 
deformation. This approach emphasized heavily on the exact and accurate recreation of the bones and body tissue to simulate the flesh deformation [4]. Due to its high demand of computation, this approach was more likely suitable for simulations requiring high degrees of accuracy e.g., crash simulations. Another approach was a physical deformation which consisted of a mass and spring system, derived from mechanical laws of particles [5]. Although this approach was less computationally demanding than the anatomical approach, it was still considered to be less suitable for real time application and was mainly for off-line simulation and animation [12]. However, the geometric approach was the cheapest in terms of computational need and hence was widely used for various applications e.g. 3D animation packages. One of the geometric approach variants commonly used was "skinning" in which the skin deformation was achieved by applying different weights to the skin vertices before undergoing rigid transformation driven by movement of the skeleton. The major drawbacks of this approach were the lack of muscle bulging/swelling and collapsing joints for extreme positions which required user intervention to correct them. Several studies were directed to overcome these problems which unfortunately increased the complexity and lessened the appeal of the skinning method itself [6], [7], [8].

A recent development in deformation methods was the example based approach [9], [10]. This method involved training the skinning model by setting the weights such that they provided the closest possible geometry to a training set of example poses. The drawbacks of this approach is the more examples poses, the more complex the motion representation would be.

Shen and Thalmann [11] and Hyun et al. [12] proposed a different approach i.e. the sweep based approach, which was characterized by the usage of cross sections to reconstruct and express the deformation. Hyun et al [12] adopted this approach by using ellipses which fit tightly to the cross section and sweep them along the link between the joints. These ellipses were then used to govern the deformation by changing its orientation, accordingly to the joint angle changes. The advantage of using this approach is its ability to preserve the volume when no self intersection happens. However, due to the collision detection algorithm, the interactive speed is slow and user intervention is sometimes needed while blending the body segments together.

Based on the review of the advantages and disadvantages of the existing approaches above, it was shown that the existing approaches focused largely on one of two areas: (i) the representation of a visually convincing human model, or (ii) the representation of an anatomically correct human model. Both of these approaches have issues either with the need for user intervention to adjust the model to achieve a satisfying result or, exhibit a high computation cost, making them unsuitable for real time use.

Supported by a TC2-NX12 3D body scanner, this research has developed a new method of flesh deformation around the joint, with the elbow as a starting point, that was automatic (minimum user intervention), realistic (visually) and accurate (dimensionally). This paper addresses how the flesh deformation method could be applied for people with different anthropometric characteristics with minimum user interventions. This feature was essential because the flesh deformation method was specially aimed for ergonomics applications which demand flexibility in simulating humans with diverse anthropometric characteristics. Further details of the flesh deformation 
method can be found in Hermawati and Marshall [13]. A brief outline of the method follows.

\subsection{Flesh Deformation Method}

In order to deform the flesh around the elbow in any posture, the proposed method required an establishment of five cross sections and a profile of the arm at the so called "key postures". The key postures were the arm in a full extension, $135^{\circ}$ flexion, $90^{\circ}$ flexion and maximum flexion.
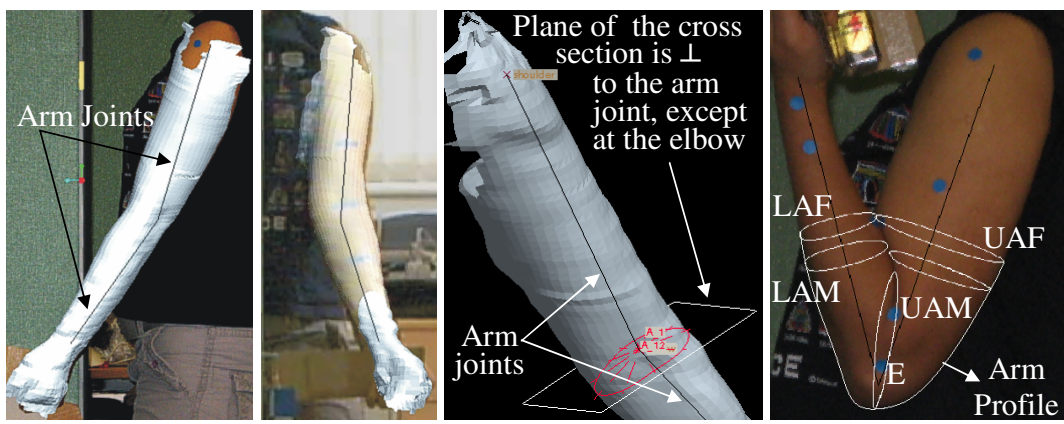

Fig. 1. Matching a 3D arm scan data with the photograph of the arm from sagittal-coronal plane and locations of the five cross sections for a fully flexed arm

The five cross sections' locations were determined by the flesh deformation area i.e., the area bounded by where the lower and upper arm met while the arm assumed a maximum flexion (see Fig. 1). These cross sections were centered at the skeleton and lay on the plane which was perpendicular to the skeleton, except for the elbow's plane which constantly changed according to the level of the arm's flexion.

The arm profile in the sagittal plane was also obtained for each key posture. The far right image of Fig. 1 shows an example of arm profile for a fully flexed arm. This

\section{KEY POSTURES}

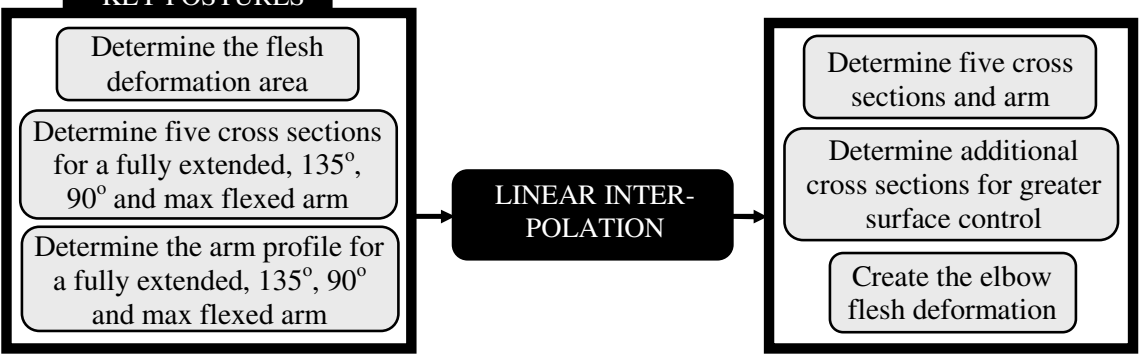

Fig. 2. The proposed flesh deformation around the elbow joint 
information was then combined to produce the elbow flesh deformation at any flexion angle by means of linear interpolation. The process is shown in the Fig. 2.

\subsection{Application of the Flesh Deformation Method for Wider Anthropometrical Range}

To enable the current method's application to simulate flesh deformation for a wide anthropometric range, the flesh deformation area, the key posture's cross sections and arm profiles had to be predicted. Five inputs are required i.e. 3D arm scan in a fully extended supine posture, height, weight, race and gender. The whole process is illustrated in Fig. 3. A relationship between the flesh deformation area and body type/size is employed to predict the flesh deformation area of a 3D arm scan. A template, which is created from a predetermined $3 \mathrm{D}$ scan of a fully extended arm, is matched to the new 3D arm scan. Using the information from the predicted flesh deformation area, five cross sections are sampled through template matching.

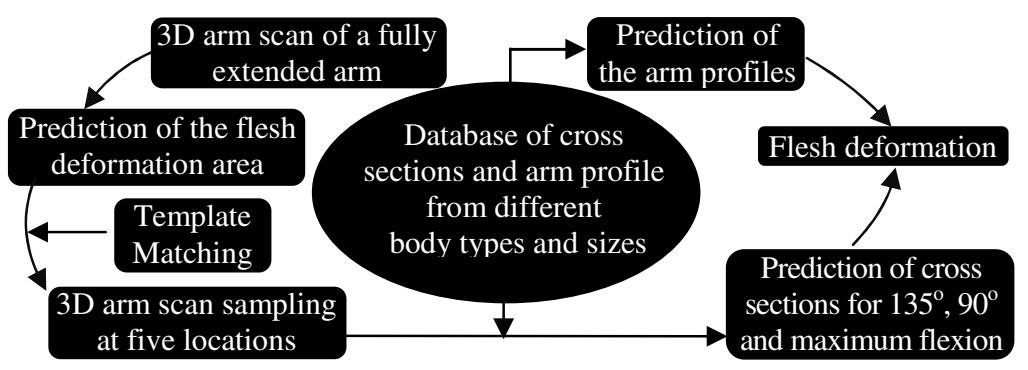

Fig. 3. Application of the flesh deformation method for wider anthropometrical range

To guide the deformation, a subject from the database that resembles closely to the sampled cross sections had to be established. The database stores cross sections information of all key postures from a range of subjects $(n=23)$ i.e. 260 cross sections altogether. Since the sampled cross sections assume full extension, only the cross sections of the fully extended arm from the database were required to determine the nearest subject. Then, the remaining data in the database from this subject i.e., cross sections for $135^{\circ}, 90^{\circ}$ and maximum flexion, were used as a basis to predict $135^{\circ}, 90^{\circ}$ and maximum flexion cross sections for the $3 \mathrm{D}$ arm scan.

\section{Methodology}

Twenty three subjects took part in this study and were grouped into 4 categories i.e. Asian females, Asian males, Caucasian males and Caucasian females. Table 1 shows the mean and standard deviation of height, weight and BMI for each group.

While the subject assumed a maximum flexion posture, markers were attached to the area where the lower and upper arm met and the distance of these markers to the elbow crease were recorded as they changed for each key posture. Then, a physical measurement to capture the changes of cross sections in four key postures by circling 
a flexible wire around the arm was carried out. The physical measurement was employed as an alternative approach of capturing the flesh deformation because of the inability of the $3 \mathrm{D}$ body scanner to capture the detail of the flesh deformation for an arm flexion $\geq 100^{\circ}$. Five cross sections were obtained during the physical measurement. The location of the cross sections were: 1) on the marker for the upper arm, 2) on the marker for the lower arm, 3) on the elbow/joint, 4) on the mid section between marker-joint for the upper arm and 5) on the mid section between marker-joint for the lower arm. Once the physical measurements were completed, a series of arm photographs with various flexion angles were taken from both the sagittal and the coronal plane. The data collection was finished by scanning the subject's arm with the 3D body scanner.

Table 1. The mean and standard deviation of height, weight and BMI for each group

\begin{tabular}{|c|c|c|c|c|}
\hline & $\begin{array}{c}\text { Asian females } \\
(\mathrm{n}=6)\end{array}$ & $\begin{array}{c}\text { Asian males } \\
(\mathrm{n}=5)\end{array}$ & $\begin{array}{c}\text { Caucasian Female } \\
(\mathrm{n}=6)\end{array}$ & $\begin{array}{c}\text { Caucasian Males } \\
(\mathrm{n}=6)\end{array}$ \\
\hline Height $(\mathrm{cm})$ & $155 \pm 7.27$ & $169.75 \pm 7.08$ & $164.83 \pm 9.13$ & $180 \pm 5.99$ \\
\hline Weight $(\mathrm{kg})$ & $57.53 \pm 14.88$ & $62.37 \pm 8.42$ & $69.57 \pm 21.5$ & $82.35 \pm 10.61$ \\
\hline BMI & $23.86 \pm 6.02$ & $22.1 \pm 3.23$ & $25.31 \pm 6.81$ & $25.48 \pm 4.74$ \\
\hline
\end{tabular}

The first step of the data processing was to determine the orientation of the upper arm joint by matching the 3D arm scan to the coronal-sagittal photographs. The joint was automatically generated by the 3D body scanner. With the help of the markers, the lower arm joint was created. This process was then followed by identifying parameters for the flesh deformation area of each key posture. For each key posture, four parameters were required i.e., the two farthest points of the flesh deformation area of both the upper (upper arm farthest-UAF) and lower arm (lower arm farthestLAF) as well as another two points between UAF/LAF and the elbow joint (upper arm middle-UAM, lower arm middle-LAM). In the initial anatomical posture, UAM and LAM was approximately located in the middle of UAF/LAF. As the UAF and LAF changed its position in respect with the elbow joint, so did the UAM and LAM. Fig. 1 shows the result of matching the 3D arm scan and the locations of five cross sections for maximum flexion.

Digitized and traced cross sections were orientated, positioned and adjusted manually, assisted by the 3D arm scans and the photographs. 16 points were sampled for every cross section and their distances towards the cross section center were calculated $\left(\delta_{1 \ldots 16}\right)$. The same distances were also determined for the template cross sections. For each cross section, its distance differences with its corresponding template was computed e.g., $\left[\Delta_{\mathrm{UAF}, 135^{\circ}}{ }^{\circ} \mathrm{UAF}_{135}{ }^{\circ}\left(\delta_{1 \ldots 16}\right)-\mathrm{UAF}_{\text {template }}\left(\delta_{1 \ldots 16}\right)\right]$. The results were stored in the database as shown in Table 2 where $\mathrm{N}$ is the total number of subjects. To compress the database size, Principal Component Analysis was applied. All data processing, apart from digitization and tracing which were processed with Corel Trace, were completed on Pro-Engineer WildFire 4.0. The flesh deformation area prediction and the cross section prediction algorithm were performed with Matlab. 
Table 2. Data arrangement in the database

\begin{tabular}{|c|c|}
\hline Row 1 & $\Delta^{\text {subjectl }}$ UAF, full extension, $\Delta^{\text {subjectl }}$ UAF, $135^{\circ}, \Delta^{\text {subjectl }}$ UAF, $90^{\circ}, \Delta^{\text {subjectl }}$ UAF,max \\
\hline Row N & $\Delta^{\text {subjectN }}{ }_{\text {UAF, full extension }}, \Delta^{\text {subjectN }}{ }_{\text {UAF, } 135^{\circ}}, \Delta^{\text {subjectN }}{ }_{\text {UAF, } 90^{\circ},}, \Delta^{\text {subjectN }}$ UAF, max \\
\hline Row Nx5 & $\Delta^{\text {subjectN }}{ }_{\text {LAF,full extension },} \Delta^{\text {subjectN }}{ }_{\text {LAF. } 135^{\circ},}, \Delta^{\text {subjectN }}{ }_{\text {LAF,90 } 90^{\circ}, \Delta^{\text {subjectN }}}{ }_{\text {LAF,max }}$ \\
\hline
\end{tabular}

\section{Results}

\subsection{Flesh Deformation Area Prediction}

Data analysis was performed separately for each group to account for the effect of race and gender. To simplify the prediction process, once the predicted UAF location was established, it was then used to generate the LAF location. This decision was based on the finding that the relationship between the UAF and LAF locations could be represented linearly as shown in Fig. 4. For each key posture, UAM and LAM locations were expressed as a fraction of UAF and LAF, respectively, and were influenced by the arm angle. As an example, Fig. 5 shows the relationship between UAF and UAM for Asian female group.

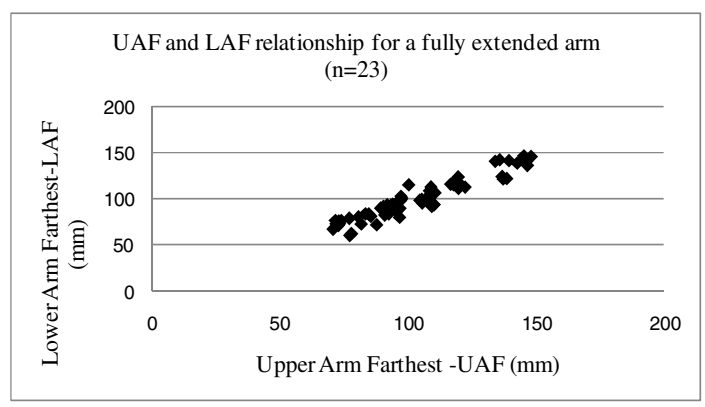

Fig. 4. Linear relationship between UAF and LAF

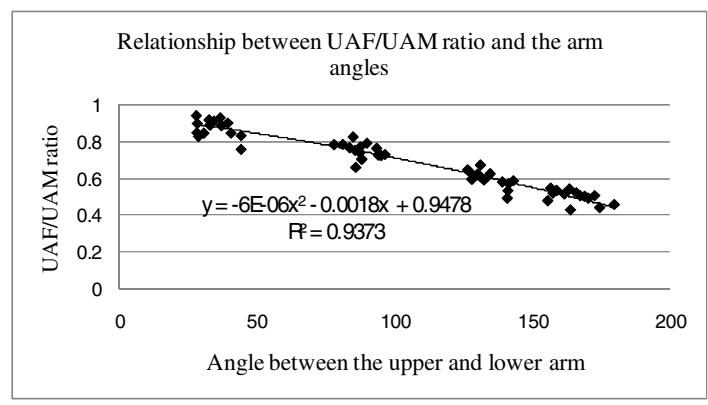

Fig. 5. A relationship between the ratio of UAF/UAM and the arm angles 
Two parameters, upper arm length and BMI, were utilized to predict the location of UAF by means of multiple regression. These parameters were chosen to represent the effect of different body size and body type on the flesh deformation area. The error, the maximum of the absolute value of the deviation of the data from the model, was shown in Table 4. Once the UAF/UAM for both full extension and flexion were found, the location of UAF/LAF was computed. The entire process for the prediction of the flesh deformation area was shown in Fig. 6.

Table 3. The absolute value of the UAF location error for a fully extended and flexed arm

\begin{tabular}{|l|c|c|c|c|}
\hline UAF Error (mm) & Asian Females & Asian Males & Caucasian Females & Caucasian Males \\
\hline Maximum extension & 12.7957 & 10.421 & 22.8327 & 15.2706 \\
\hline Maximum flexion & 3.9011 & 1.7325 & 9.0095 & 13.3917 \\
\hline
\end{tabular}

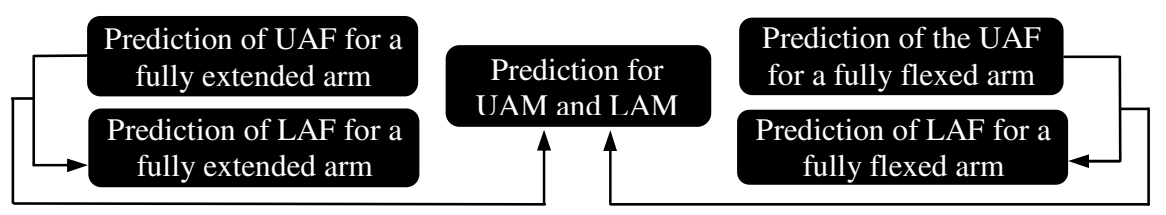

Fig. 6. Entire process of the flesh deformation area prediction

The flesh deformation area prediction was tested on three new data i.e., one Asian female, one Asian male and one Caucasian male. Table 4 showed the errors for each test data.

Table 4. Euclidian distance error of flesh deformation area prediction for three data test

\begin{tabular}{|l|c|c|c|c|c|c|}
\hline & \multirow{2}{*}{ BMI } & \multirow{2}{*}{$\begin{array}{l}\text { Height } \\
(\mathrm{cm})\end{array}$} & & \multicolumn{4}{|c|}{ Error (mm) } \\
\cline { 4 - 7 } & & & \multicolumn{2}{|c|}{ Maximum extension } & Maximum flexion \\
\cline { 4 - 7 } & & & Upper arm & Lower arm & Upper arm & Lower arm \\
\hline Asian female & 20.45 & 165 & 0 & 2.57 & 2.68 & 2.58 \\
\hline Asian male & 22.03 & 170 & 23.89 & 20.8 & 10.4 & 7.83 \\
\hline Caucasian male & 23.33 & 177 & 0.53 & 0 & 4.16 & 7.99 \\
\hline
\end{tabular}

\subsection{Cross Sections' Prediction}

A template was matched to the 3D arm scan of a fully extended arm to obtain the cross sections at five locations. To overcome frequent occurrence of noisy and missing data around the apex of the 3D arm scan, which otherwise affected the sampling outcome of UAM and UAF, sagittal and coronal profiles of the 3D arm scan were used to correct the UAM and UAF cross sections automatically.

To simplify the cross section prediction process, all cross sections were transformed into a 2 dimensional coordinate system. For the cross section prediction, gender differences were overlooked since an earlier observation demonstrated the irrelevance of gender on the shape of the cross sections. Three different methods were applied to seek the best matched subject from the database. Because the 
sampled cross sections were required from a fully extended 3D scan arm, only the cross sections of the fully extended arm from the database were required to determine the best matched subject.

For the upper part of the elbow, the relationship between UAF-UAM was employed to find the best matched subject from the database. The cross section changes of the best matched subject were applied on the new 3D arm scan and followed by a scaling adjustment to accommodate for cross sections' size difference. The relationship between UAM and UAF was defined as:

$$
\begin{aligned}
& \text { Y_ratio }=\frac{(\text { maximum UAF y value- minimum UAF y value })}{(\text { maximum UAM y value- minimum UAM y value })} . \\
& X \text { _ratio }=\frac{(\text { maximum UAF } x \text { value- minimum UAF x value })}{(\text { maximum UAM x value- minimum UAM x value })} .
\end{aligned}
$$

For the lower part of the elbow and the elbow itself, Principal Component Analysis (PCA) was utilized to determine the best matched subject from the database. PCA was useful to summarize features which distinguished cross sections from one subject to another. PCA was applied for E and LAM cross sections after subtracting them with the corresponding template cross sections. Matching was performed by comparing PCA values for LAM. The subject with the closest PCA value in the database to the target value was selected. Once the closest matched subject for LAM was found in the database, the LAF cross sections would be extracted from the same subject. A scaling adjustment to accommodate for cross sections size difference was also applied for the prediction of the lower part of the elbow.

The elbow cross section prediction was in a way similar as that of the prediction for the lower part of the elbow. However, instead of using one subject to guide the prediction of the cross sections, a linear interpolation between two closest subjects was employed. This step was necessary because the elbow joint shape was much more complex than that of the lower part of the elbow.

The cross sections prediction was validated by comparing them with either cross sections of a 3D scan arm at available key posture or the photographs. The comparison with the $3 \mathrm{D}$ scan arm was mostly available for $135^{\circ}$ flexion and in some occasion for $90^{\circ}$ flexion (the upper part of the elbow only). For each cross section, the Euclidean distance difference between the predicted cross section and the 3D arm cross section were computed at 32 points to obtain the maximum absolute deviation and the

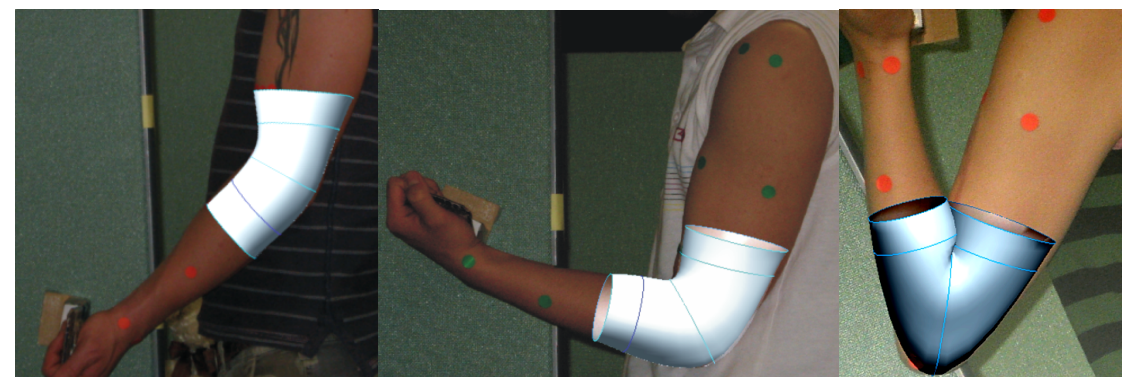

Fig. 7. The outcome of flesh deformation and cross sections prediction at three key postures of three different data test 
deviation average. The maximum absolute deviation was $\pm 5.46 \mathrm{~mm}$ and the average of the deviation was $\pm 1.96 \mathrm{~mm}$.

Images of the outcome of the flesh deformation and the cross sections prediction is shown in Fig. 7.

\section{Discussion}

The validation of flesh deformation area prediction shows a mixed outcome regarding its level of accuracy. This might be caused partly by such a small number of samples in this study. With a sample of $\approx 6$ people in each group, there is a possibility that the true relationship between BMI, upper arm size and flesh deformation area is not correctly represented. In addition to this, the flesh deformation area is largely affected by arm muscularity that neither the BMI nor the upper arm length properly capture. Hence, there is a strong likelihood that additional parameters are required e.g. body type, skin fold measurement. Another factor which might cause the mixed outcome is the fact that, in this study, the flesh deformation area was defined while the participants assumed a fully flexed elbow. This meant that the range of motion of the elbow flexion would also influence the flesh deformation area and yet, for simplicity, a uniform range of elbow flexion was applied during data analysis and prediction. In addition to this, no correlation between the elbow range of motion and BMI was made despite a study finding [14], which showed BMI was negatively correlated with the elbow flexion range.

The small number of available data in the database might also cause the relatively low accuracy of the cross sections predictions. Since the represented feature of the cross sections in the database was limited, it might lead to an incorrect choice of the best matched subject for the cross section prediction. In addition to this, the combination of physical measurement and 3D arm scan data usage might be another factor which contributed to the low accuracy of the cross sections predictions although much care was taken to ensure the accuracy of the physical measurement by introducing the photographs for cross sections refinement. Nonetheless, even with such a low number of data in the database to refer to, the result of this method demonstrated a potential for a further development. This method could also be used for other simple joint such as the knee. However, a complexity might arise for an application on a complex joint such as the shoulder. In this study, even though there were only 23 participants involved, the amount of work that was undertaken to gather and analyze their data was quite substantial. This might be seen as a drawback for a wider study. However, it might be no longer a problem once the 3D body scanning capability has improved e.g. marker detection ability, better handling for data occlusion, better accuracy for joint allocation etc.

The next stage of the study is to analyze the arm profiles from the collected data and then establishing the prediction method of the arm profile for any 3D scan arm input. This step will be the last stage of the research and the overall validation for both the flesh deformation method and the flesh deformation prediction would then be validated.

\section{Conclusions}

A flesh deformation model, that can represent the deformation of the flesh deformation at the elbow join, has been developed. The flesh deformation area and cross 
section prediction showed a positive result regarding its accuracy despite being based on a limited range of anthropometric data. Furthermore, the prediction was acquired without user intervention. The prediction result accuracy could be improved further by gathering a wider anthropometric data range. This flesh deformation prediction fulfilled the identified ergonomics application requirements i.e., accurate, no user intervention and accommodating various anthropometric parameters.

\section{References}

1. Locket, J.F., Assmann, E., Green, R., Reed, M.P., Rascke, R., Verriest, J.P.: Digital Human Modeling Research and Development User Needs Panel. In: Proc. SAE Digital Human Modelling for Design and Engineering Symposium. SAE, Iowa City (2005)

2. Wegner, D., Chiang, J., Kemmer, B., Lämkull, D., Roll, R.: Digital Human Modeling Requirements and Standardization. In: Proc. of Digital Human Modeling for Design and Engineering Conference and Exhibition. SAE, Seattle (2007)

3. Lämkull, D., Hanson, L., Örtengren, R.: The Influence of Virtual Human Model Appearance on Visual Ergonomics Posture Evaluation. Applied Ergonomics 38, 713-722 (2007)

4. Dong, F., Clapworthy, G.J., Krokos, M.A., Yao, J.: An Anatomy-Based Approach to Human Muscle Modeling and Deformation. IEEE Transaction on Visualization and Computer Graphics 8, 154-170 (2002)

5. Vassilev, T., Spanlang, B.: A Mass-Spring Model for Real Time Deformable Solid. EastWest-Vision (2002)

6. Mohr, A., Tokheim, L., Gleicher, M.: Direct Manipulation of Interactive Character Skins. In: Proc. of the symposium on Interactive 3D graphics, pp. 27-30. ACM, New York (2003)

7. Kavan, L., Žảra, J.: Spherical Blend Skinning: A Real-Time Deformation of Articulated Models. In: Proc. of the 2005 symposium on Interactive 3D graphics and games, pp. 9-16. ACM, New York (2005)

8. Mohr, A., Gleicher, M.: Building Efficient, Accurate Character Skins From Examples. In: Proc. of International Conference on Computer Graphics and Interactive Techniques, pp. 562-568. ACM, New York (2003)

9. Allen, B., Curless, B., Popovic, Z.: Articulated Body Deformation From Range Scan Data. In: Proc. of the 29th annual conference on Computer graphics and interactive techniques, pp. 612-619. ACM, New York (2002)

10. Wang, X.C., Phillips, C.: Multiweight Enveloping: Least Square Approximation Techniques for Skin Animation. In: Proc. of the 2002 ACM SIGGRAPH/Eurographics Symposium on Computer Animation, pp. 129-138. ACM, New York (2002)

11. Shen, J., Thalmann, D.: Realistic Human Body Modeling and Deformation. Master Thesis, Ecoles Polytechniques fédérale de Lausanne (1996)

12. Hyun, D.E., Yoon, S.H., Chang, J.W., Seong, J.K., Kim, M.S., Jüttler, B.: Sweep-based Human Deformation. The Visual Computer 21, 542-550 (2005)

13. Hermawati, S., Marshall, R.: Realistic Flesh Deformation for Digital Humans in Ergonomics Modeling. In: Proc. Digital Human Modeling for Design and Engineering Symposium. SAE, Pittsburgh (2008)

14. Golden, D., Wojcicki, J., Jhee, J.T., Gilpin, S.L., Sawyer, J.R., Heyman, M.B.: Body Mass Index and Elbow Range of Motion in a Healthy Pediatric Population. Journal of Pediatric Gastroenterology and Nutrition 46, 196-201 (2008) 